\title{
The importance of MLH1, MSH2, MSH6 and PMS2 in determining DNA damage response in digestive cancers
}

\author{
FLAVIU PITU ${ }^{1,2}$, SERGIU PASCA ${ }^{1}$, CALIN POPA ${ }^{3}$, AL HAJJAR NADIM ${ }^{1,3}$ \\ 1"Iuliu Hatieganu" University of Medicine and Pharmacy, Faculty of Medicine, Dept. of General \\ Surgery, Cluj-Napoca, Romania \\ ${ }^{2}$ Surgery Department, Alba Iulia County Emergency Hospital, Bld. Revolutiei 1989 No. 23, Alba \\ Iulia, Romania \\ ${ }^{3}$ Regional Institute of Gastroenterology and Hepatology “Octavian Fodor”, Cluj-Napoca, Romania
}

\begin{abstract}
Introduction: Genomic instability is a common feature across human cancers, but presents variation across different types of cancer, patients and cells of from the same tumor.This can be caused either by changes in the DNA damage repair pathways, aberrant histone modifications or methylation. The aim of this study was to determine the common mutated genes between digestive cancers that present genomic instability and to determine the biological processes in which these are implicated.

Material and Methods: Mutational profiles for patients presenting digestive cancers and mutations in MLH1, MSH2, MSH6 and PMS2 were downloaded from the MSK impact cohort via cBioPortal. PANTHER was used to determine the GO SLIM processes in which the mutated genes are involved. R 3.5.3 and the R package circlize were used to generate chord diagrams.

Results and Discussions: Considering the number of mutated genes from different digestive cancers it could be observed that the deregulated processes are general cellular processes, while when taking into consideration the fold change in overrepresentation of certain processes, the main deregulated processes are represented by DNA damage repair pathways, showing their overrepresentation in the selected cohort based on a few mutated genes.

Conclusion: MLH1,MSH2,MSH6 and PMS2 mutations dictate the alterations in DNA damage repair pathways in digestive cancers.
\end{abstract}

Keywords Digestive cancers, genomic instability, immunotherapy.

To cite this article: PITU F, PASCA S, POPA C, NADIM AH. The importance of MLH1, MSH2, MSH6 and PMS2 in determining DNA damage response in digestive cancers. Rom Biotechnol Lett. 2021; 26(1): 2289-2294. DOI: 10.25083/rb1/26.1/2289.2294

$\triangle$ *Corresponding author: Corresponding author: PITU FLAVIU, MD, Ph-D, Surgery Department, Alba Iulia County Emergency Hospital, Bld. Revolutiei 1989 No. 23, Alba Iulia, Romania E-mail: pitu.flaviu@umfcluj.ro Tel.: 0744504079 


\section{Introduction}

Genomic instability is a common feature across human cancers, but presents variation across different types of cancer, patients and cells of from the same tumor. This can be caused either by changes in the DNA damage repair pathways, aberrant histone modifications or methylation (1-3). One clinically applied method for determining the grade of genomic instability is through assessing the grade of microsatellite instability, which was first applied to colon cancer (4). An indirect way of assessing microsatellite instability is through immunohistochemical staining for proteins implicated in DNA damage repair. At first, MLH1 and MSH2 were used, later MSH6 and PMS2 being added, showing high sensitivity and specificity to detecting the microsatellite instability grade (5-8).

Genomic instability can create oncogenes and inactivate tumor suppressors, but it also creates neoantigens, which are better detected by the immune system. Because of this, malignant cells presenting high genomic instability and high number of neoantigens will better respond to immunotherapy $(9,10)$. The first malignancy that benefitted from this was melanoma, in which checkpoint inhibitors offered a better prognosis (11). After this first application, checkpoint inhibitors continued to be applied in other cancers reaching the current guidelines, which specify allow for the use of pembrolizumab in solid tumors if high MSI is proven for the patients malignancy (12).

The importance if immune interactions does not show importance only in therapy choice, but also in prognosis, immune classifications of colon cancer with good prognosis prediction appearing more and more lately, showing the importance of the immune system and it's applicability in the field of oncology (13). Because colon cancer is also assessed through MSI status and because of the frequency that digestive cancer present (14), we chose to expand the genomic instability term to other cancers from the same field of digestive oncology. The aim of this study was to determine the common mutated genes between digestive cancers that present genomic instability and to determine the biological processes in which these are implicated.

\section{Material and Methods}

\section{Patient selection}

The patients included in the present study were selected from the MSK Impact cohort (15). The online data was downloaded via cBioPortal $(16,17)$. We included the following cancer types: colon adenocarcinoma, colorectal adenocarcinoma, rectal adenocarcinoma, stomach adenocarcinoma, esophagogastric adenocarcinoma, esophageal adenocarcinoma, pancreatic adenocarcinoma, cholangiocarcinoma that also presented mutations in MLH1, MSH2, MSH6 or PMS2.

\section{Data analysis}

Data analysis was performed using $\mathrm{R}$ 3.5.3 and PANTHER (18). We included only the GO SLIM processes that had a false discovery rate (FDR) under 0.05 . Chord diagrams were generated using the circlize R package (19).

\section{Results and Discussions}

After applying the inclusion criteria we included in the study 85 patients with a total of 88 samples. Of the samples, 64 were collected from the primary site and 24 from the metastatic site. Of the 64 primary sites, 43 were CRAD (colorectal adencarcinoma), defined as colon/ rectum or colorectal adenocarcinoma, 11 were EGAD (esophagogastric adenocarcinoma), defined as gastric/ esophageal or esophagogastric adenocarcinoma, 7 were PAD (pancreatic adenocarcinoma), defined as pancreatic adenocarcinoma and 3 were ChC (Cholangiocarcinoma), defined as either intra- or extrahepatic cholangiocarcinoma. Of the 24 metastatic sites, 17 were CRAD, 4 were EGAD, 2 were $\mathrm{ChC}$ and 1 was PAD.

After sample selection, we analyzed the distinct gene mutations that occurred in primary and metastatic site samples and between different tumor types. We introduced the distinct mutated genes by cancer and site in PANTHER and assessed the overrepresentation of certain GO SLIM processes. Chord diagrams were generated to offer the overview of the shared biological processes. The width of the links in chord diagrams was either the number of mutated genes in the process or the fold change in overrepresentation and in each case, we only included in the chord diagram processes in which there were either more than 20 changed genes from our list or there was a fold change of more than 20 .

The tables presenting the GO SLIM biological processes for primary and metastasis site can be fount in Supplementary Table 1 and Supplementary Table 2 respectively.

Figure 1 was generated taking the genes mutated in the primary site and compare them across cancers considering the number of genes implicated in that process. PAD was not included because of the low number of distinct mutated genes per process. 
The following processes were observed to be common between CRAD, ChC and EGAD: (GO:0009987), biological regulation (GO:0065007), regulation of biological process (GO:0050789), cellular response to stimulus (GO:0051716). Through this assessment, the majority of processes are shared between CRAD and EGAD showing either common processes probably because of similar deregulated processes or because of overrepresentation of these cancer types in the cohort and the presence of a higher number of possible mutated genes.

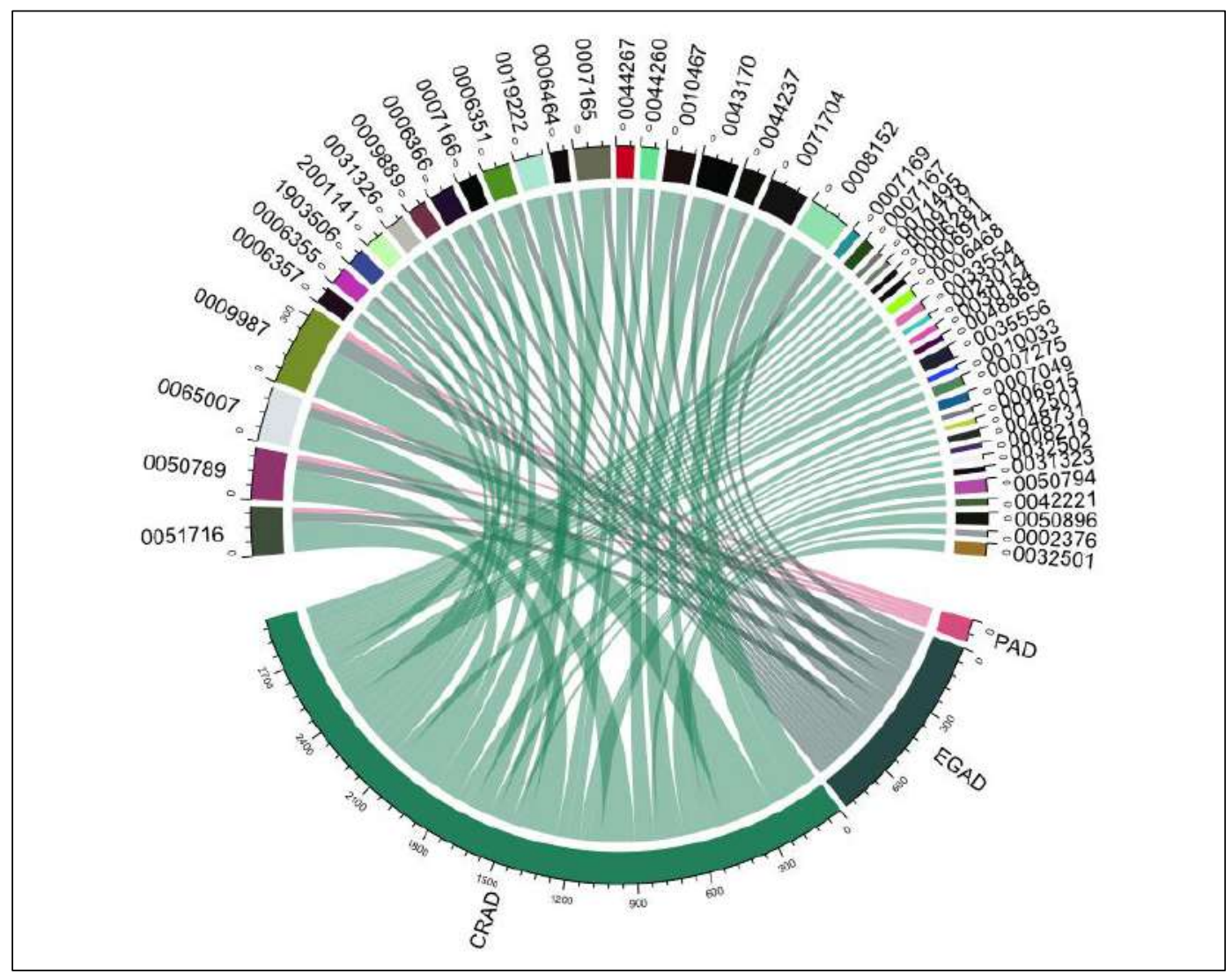

Figure 1. Chord diagram of GO SLIM processes in primary site samples considering the number of mutated genes as the width of the links.

Figure 2 was generated taking the genes mutated in the primary site and compare them across cancers considering the fold change of the genes implicated in that process. The process common among CRAD, EGAD and $\mathrm{ChC}$ with the highest fold change enrichment was is mismatch repair (GO:0006298), which shows the importance of MLH1, MSH2, MSH6 and PMS2 in regulating DNA damage response pathways. CRAD and EGAD overlap in most processes, the highest fold change being represented by mitotic G2 DNA damage checkpoint (GO:0007095). ChC only one process overlaps with PAS, being represented by mismatch repair (GO:0006298) and, in the case of, PAD only 2 processes overlap with CRAD, being represented by sprouting angiogenesis (GO:0002040) and protein kinase B signaling (GO:0043491), showing that these two cancers can benefit from VEGF inhibitors of protein kinase inhibitors, alongside checkpoint inhibitors. In PAD the highest fold change process that is present only in this type of cancer is represended by interstrand cross-link repair (GO:0036297).

Figure 3 was generated taking the genes mutated in the metastatic site and compare them across cancers considering the number of genes implicated in that process. PAD was not included because of the low number of distinct mutated genes per process. The common process between CRAD, EGAD and ChC was biological regulation (GO:0065007). Between CRAD and EGAD there were four processes that overlapped: cellular process (GO:0009987), biological regulation (GO:0065007), cellular response to stimulus (GO:0051716), macromolecule metabolic process (GO:0043170). 


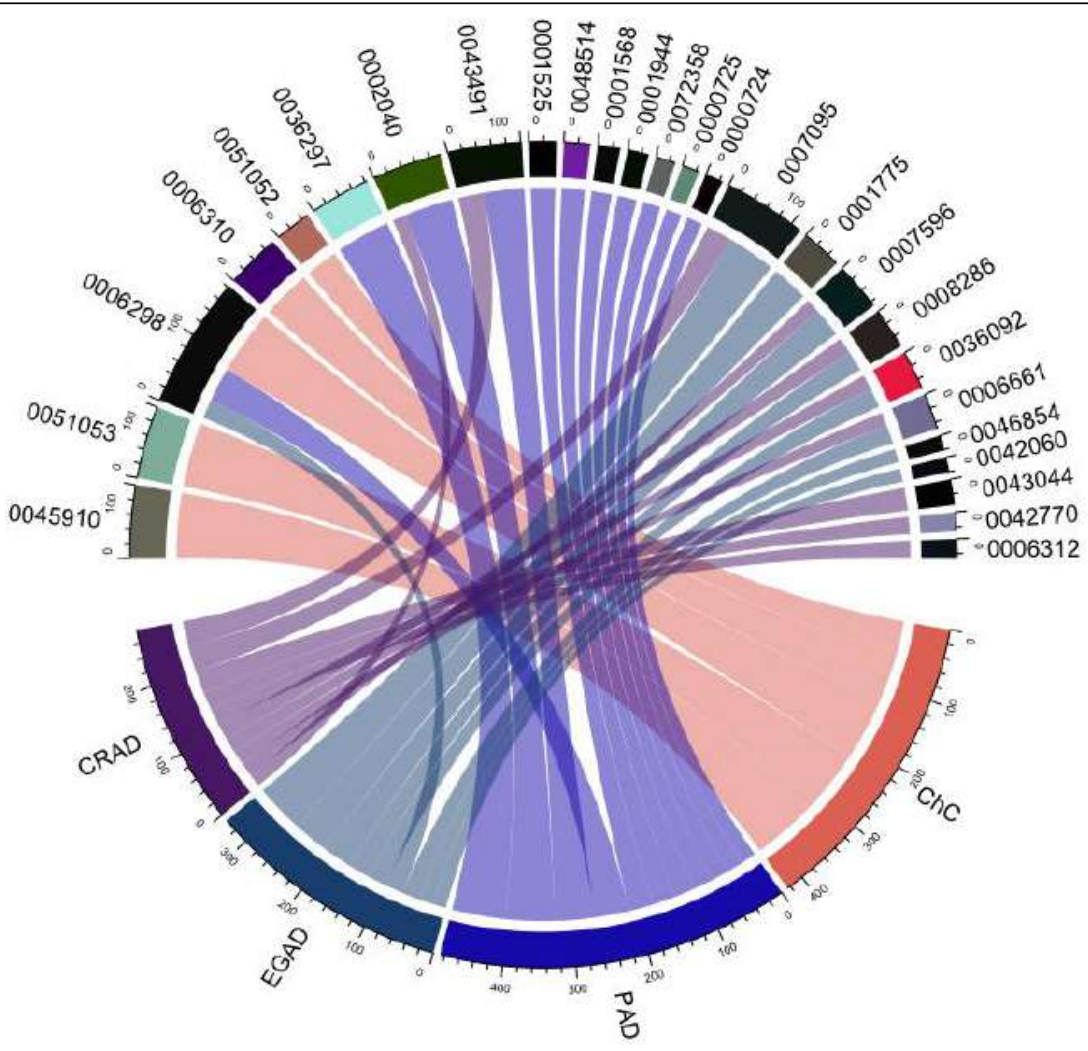

Figure 2. Chord diagram of GO SLIM processes in primary site samples considering the fold change overrepresentation as the width of the links.

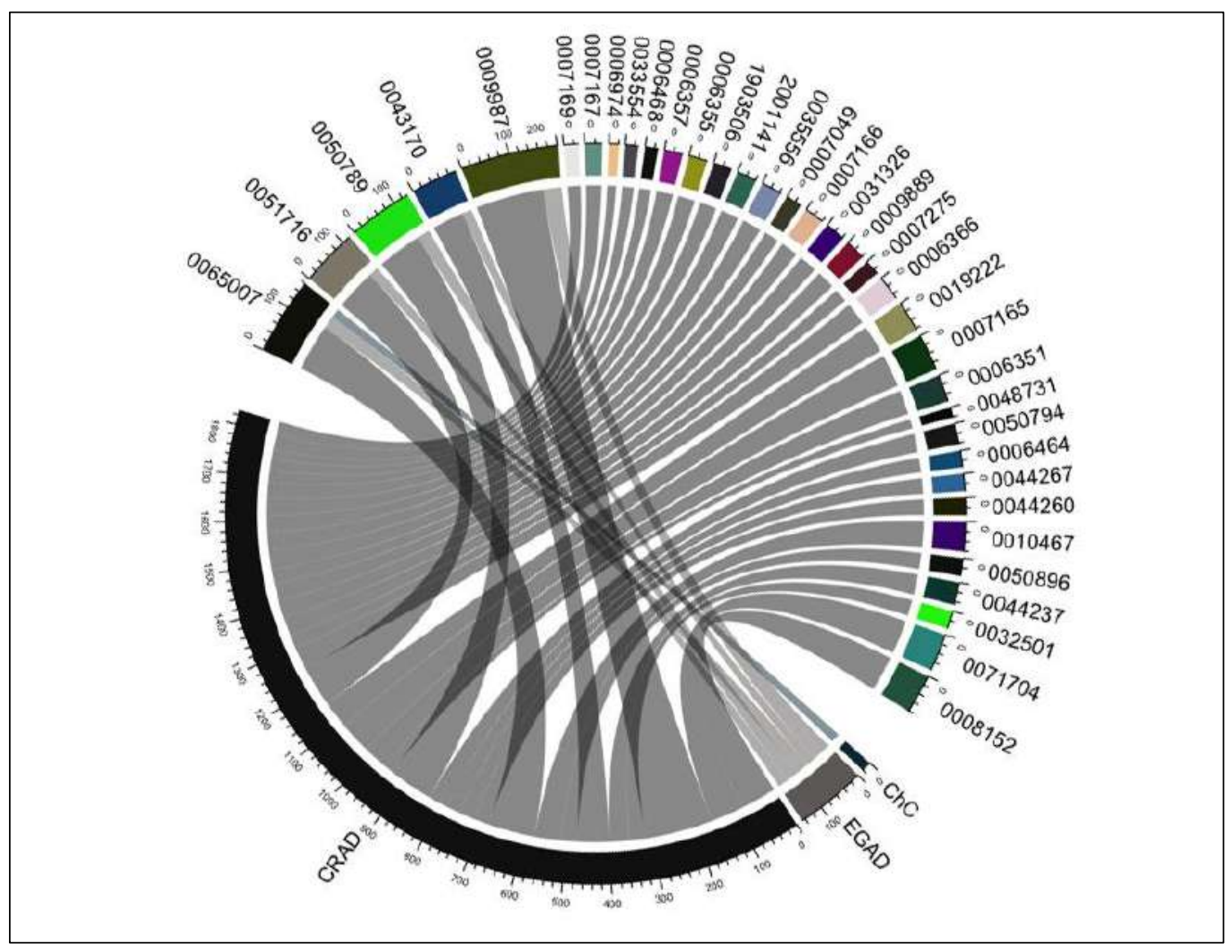

Figure 3. Chord diagram of GO SLIM processes in metastatic site samples considering the number of mutated genes as the width of the links. 
Figure 4 was generated taking the genes mutated in the metastatic site and compare them across cancers considering the fold change of the genes implicated in that process. There were two processes shared across $\mathrm{ChC}$ and CRAD, represented by mitotic G2 DNA damage checkpoint (GO:0007095), DNA damage checkpoint (GO:0000077). There was one process shared across CRAD and EGAD nucleotide-excision repair, DNA incision (GO:0033683).

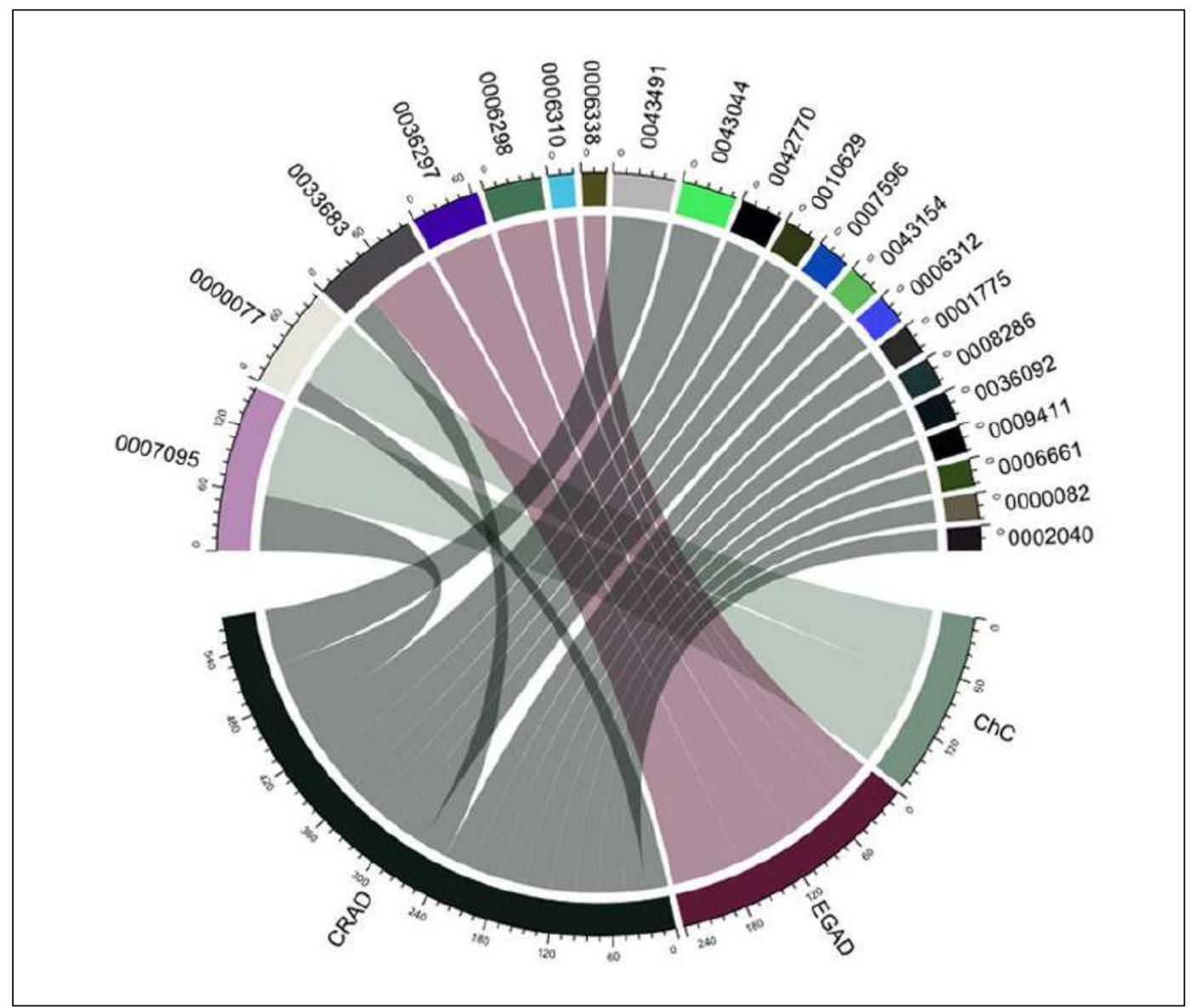

Figure 4. Chord diagram of GO SLIM processes in metastatic site samples considering the fold change overrepresentation as the width of the links.

It could be observed that most processes shared when taking into consideration the number of genes implicated in the process were general cellular processes, while, when taking into account the fold change, most shared and important processes were represented by DNA damage repair pathways, showing the importance of MLH1, MSH2, MSH6 and PMS2 mutations rather than the type of cancer in altering DNA damage repair pathways.

\section{Conclusion}

In the selected digestive cancers MLH1, MSH2, MSH6 and PMS2 mutations dictate the perturbation of DNA damage repair pathways, thus reconfirming the utility of checkpoint inhibitors in cancers that present microsatellite instability assessed indirectly through these gene mutations.

\section{References}

1. FERGUSON LR, CHEN H, COLLINS AR et al. Genomic instability in human cancer: Molecular insights and opportunities for therapeutic attack and prevention through diet and nutrition. Semin Cancer Biol. 2015;35 Suppl(Suppl):S5-S24. doi:10.1016/ j.semcancer.2015.03.005

2. YAO Y, DAI W. Genomic Instability and Cancer. J Carcinog Mutagen. 2014;5:1000165.

3. BURRELL RA, MCGRANAHAN N, BARTEK J, SWANTON C. The causes and consequences of 
genetic heterogeneity in cancer evolution. Nature. 2013 Sep 19; 501(7467):338-45.

4. PIKOR L, THU K, VUCIC E, LAM W. The detection and implication of genome instability in cancer. Cancer Metastasis Rev. 2013; 32(3-4):341-352.

5. ASAD UMAR, C. RICHARD BOLAND, JONATHAN P. TERDIMAN et al. Revised Bethesda Guidelines for Hereditary Nonpolyposis Colorectal Cancer (Lynch Syndrome) and Microsatellite Instability, JNCI: Journal of the National Cancer Institute, Volume 96, Issue 4, 18 February 2004, Pages 261-268.

6. SHIA J. Immunohistochemistry versus microsatellite instability testing for screening colorectal cancer patients at risk for hereditary nonpolyposis colorectal cancer syndrome. Part I. The utility of immunohistochemistry. J Mol Diagn. 2008;10(4):293-300.

7. JONG AE, PUIJENBROEK M, HENDRIKS Y et al. Microsatellite Instability, Immunohistochemistry, and Additional PMS2 Staining in Suspected Hereditary Nonpolyposis Colorectal Cancer. Clin Cancer Res 2004 (10) (3) 972-980.

8. BAEK DW, KANG BW, LEE SJ et al. Clinical Implications of Mismatch Repair Status in Patients With High-risk Stage II Colon Cancer. In Vivo. 2019; 33(2):649-657. doi:10.21873/invivo. 11523

9. BEVER KM, LE DT. DNA repair defects and implications for immunotherapy. J Clin Invest. 2018 Oct 1;128(10):4236-4242.

10. YAGHMOUR G, PANDEY M, IRELAND $\mathrm{C}$ et al. Role of Genomic Instability in Immunotherapy with Checkpoint Inhibitors. Anticancer Res. 2016 Aug; 36(8):4033-8.

11. LUGOWSKA I, TETERYCZ P, RUTKOWSKI P. Immunotherapy of melanoma. Contemp Oncol (Pozn). 2018; 22(1A):61-67. doi:10.5114/wo.2018.73889
12. MARCUS L, LEMERY SJ, KEEGAN P et al. FDA Approval Summary: Pembrolizumab for the treatment of microsatellite instability-high solid tumors. Clin Cancer Res 2019.

13. COHEN R, HERAN M, PUDLARZ T et al. [Going beyond microsatellite instability for immunotherapy in metastatic colorectal cancer: Consensus molecular subtypes and tumor mutational burden]. Bull Cancer. 2019 Feb; 106(2):151-161.

14. SIEGEL RL, MILLER KD, JEMAL A. Cancer statistics, 2019. CA Cancer J Clin. 2019 Jan;69(1): 7-34.

15. ZEHIR A, BENAYED R, SHAH RH et al. Mutational landscape of metastatic cancer revealed from prospective clinical sequencing of 10,000 patients [published correction appears in Nat Med. 2017 Aug 4; 23 (8):1004]. Nat Med. 2017; 23(6):703-713.

16. GAO J, AKSOY BA, DOGRUSOZ U et al. Integrative analysis of complex cancer genomics and clinical profiles using the cBioPortal. Sci Signal. 2013; 6(269):pl1. Published 2013 Apr 2. doi:10.1126/ scisignal.2004088

17. CERAMI E, GAO J, DOGRUSOZ U et al. The cBio cancer genomics portal: an open platform for exploring multidimensional cancer genomics data [published correction appears in Cancer Discov. 2012 Oct; 2(10):960]. Cancer Discov. 2012;2(5):401-404.

18. MI H, HUANG X, MURUGANUJAN A. PANTHER version 11: expanded annotation data from Gene Ontology and Reactome pathways, and data analysis tool enhancements. Nucleic Acids Research, Volume 45, Issue D1, January 2017, Pages D183-D189.

19. GU Z, GU L, EILS R et al. circlize Implements and enhances circular visualization in R. Bioinformatics. 2014 Oct; 30(19):2811-2. 